\section{Un nouveau type \\ de rejet de greffe induit par les lymphocytes natural killer: le rejet chronique vasculaire 《inné »}

Sarah Hamada ${ }^{1}$, Olivier Thaunat ${ }^{1-3}$, Alice Koenig ${ }^{1-3}$

\author{
${ }^{1}$ CIRI, Inserm U1111, Université Claude Bernard Lyon I, \\ CNRS UMR5308, École normale supérieure de Lyon, Univ. Lyon, \\ 21 avenue Tony-Garnier, 69007 Lyon, France. \\ ${ }^{2}$ Hospices civils de Lyon, Hôpital Edouard Herriot, Service \\ de transplantation, néphrologie et immunologie clinique, \\ 5 place d'Arsonval, 69003 Lyon, France. \\ ${ }^{3}$ Faculté de médecine Lyon-Est, Université Claude Bernard \\ Lyon I, 8 avenue Rockfeller, 69373 Lyon, France. \\ olivier.thaunat@chu-lyon.fr
}

Le rejet du greffon en transplantation La transplantation d'organe est l'option thérapeutique de choix (souvent la seule possible) pour les patients présentant une défaillance d'un organe vital. Malheureusement, la survie de l'organe transplanté est limitée par le rejet de l'allogreffe. Ce processus de destruction du greffon résulte de la capacité du système immunitaire du receveur à discriminer le greffon comme une cible. Le dogme actuel est que la reconnaissance des déterminants alloantigéniques principalement les molécules du complexe majeur d'histocompatibilité (CMH) spécifiques du donneur - relève exclusivement du système immunitaire adaptatif du receveur, c'est-à-dire des lymphocytes $T$ et B (Figure IA). Alors que le rejet cellulaire, impliquant les lymphocytes T, est efficacement prévenu par les traitements immunosuppresseurs actuels, il n'existe aucun traitement efficace contre le rejet impliquant les anticorps spécifiques du donneur (donor-specific antibodies). Ce rejet humoral est actuellement considéré comme la principale cause de perte des greffons [1].

\section{Bases immuno-pathologiques du rejet humoral}

Les anticorps spécifiques du donneur, du fait de leur taille, ont une capacité limitée à diffuser en dehors des vaisseaux [2]. Ils se fixent préférentiellement aux molécules allogéniques du CMH, directement accessibles, exprimées à la surface des cellules endothéliales de la microvascularisation du greffon (Figure $I A$ ). Si la quantité d'anticorps spécifiques du donneur fixés est suffisante, l'activation de la voie classique du complément conduit à une destruction rapide du greffon [3] (Figure 1A). Ce processus n'est cependant pas indispensable au développement d'un rejet d'évolution plus lente (rejet «chronique ») [4, 5]. $\varepsilon$ n effet, les anticorps spécifiques du donneur fixés aux cellules endothéliales permettent aussi le recrutement d'effecteurs de l'immunité innée, qui lèsent les cellules endothéliales du greffon en libérant des enzymes lytiques (antibody-dependent cell-mediated cytotoxicity, ADCC) (Figure 1A). Parmi les multiples cellules effectrices de l'immunité innée exprimant des récepteurs pour la région $F c$ des IgG (RFc $\gamma)$, lymphocytes natural killer (NK) et $\mathrm{T} \gamma \delta$ [6], monocytes, etc., les lymphocytes NK ont été identifiés comme des acteurs majeurs dans l'apparition des lésions d'inflammation microvasculaire $[5,7]$ considérées comme caractéristiques du rejet humoral [8] (Figure 1A).

$\varepsilon n$ étudiant un groupe de patients porteurs d'un greffon rénal, nous avons identifié certains individus qui présentaient des lésions microvasculaires d'inflammation inexpliquées, c'est-àdire survenant en l'absence d'anticorps spécifiques du donneur détectables, à l'analyse histopathologique d'une biopsie du greffon. Chez ces individus, la survie du greffon était identique à celle des patients présentant un rejet humoral chronique, et le nombre de lymphocytes NK infiltrant le greffon était également similaire [9]. Cela nous a conduits à postuler que, si le rejet chronique vasculaire implique toujours les lymphocytes NK, l'activation de ces derniers est parfois indépendante de la présence d'anticorps spécifiques du donneur.

Inflammation microvasculaire indépendante des anticorps spécifiques du donneur, déclenchée par la reconnaissance du « soi-manquant 》 (« missing self »)

Les lymphocytes NK possèdent, outre leurs récepteurs $F c \gamma$, d'autres récepteurs de surface, notamment les killer Ig-like receptors (KIR) inhibiteurs [10]. En conditions physiologiques, les KIR inhibiteurs, qui interagissent avec les molécules du CMH de classe I (CMH-I) du soi présentes à la surface des cellules saines environnantes, délivrent des signaux prévenant l'activation des lymphocytes NK. La diminution de l'expression des molécules du CMH-I par les cellules tumorales ou infectées par un virus induit une levée d'inhibition permettant aux lymphocytes NK de lyser ces cibles: un processus connu sous le nom de reconnaissance du «soi-manquant» (en anglais, « missing self »).

$\varepsilon n$ transplantation, donneur et receveur sont génétiquement différents, 


\section{ALLORECONNAISSANCE ADAPTATIVE}

Cellule dendritique du donneur Cellule dendritique du receveur

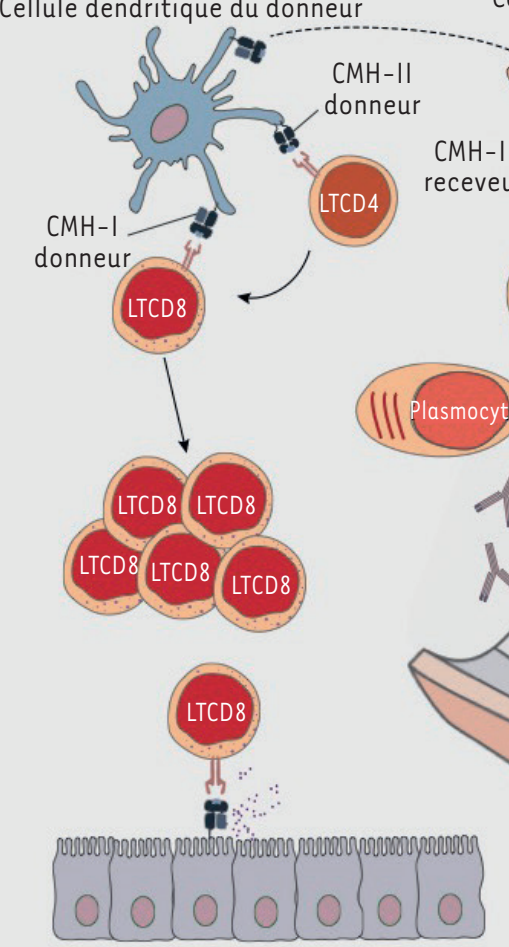

Cellules tubulaires du greffon

$\downarrow$

Inflammation interstitielle

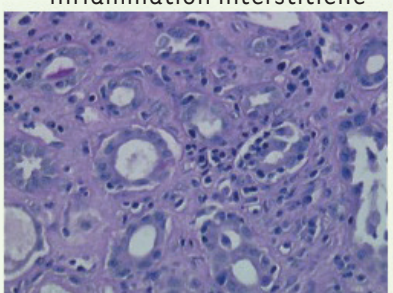

Tubulite

LB

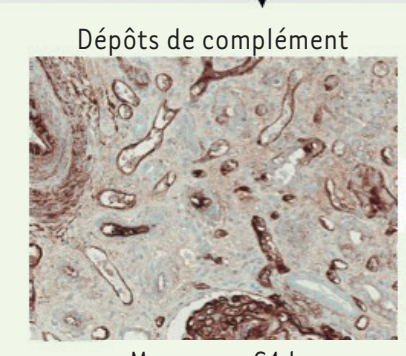

Marquage $C 4 \mathrm{~d}$
Mot

RejeTHUC

REJET HUMORAL

\section{B}

\section{ALLORECONNAISSANCE INNÉE}

Cellules du receveur
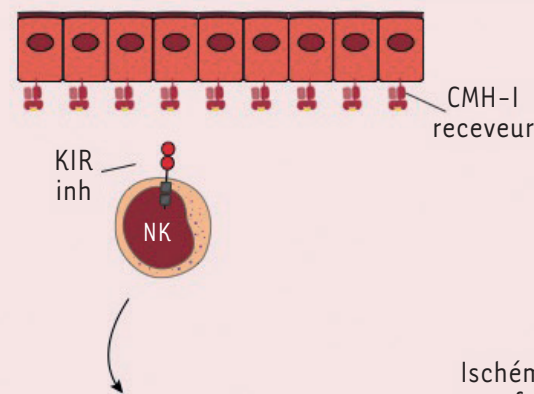

receveur

REJET CELLULAIRE

REJET PAR LES LYMPHOCYTES NK INDUIT PAR MISSING SELF

Figure 1. Physiopathologie des rejets de greffe « adaptatifs 》 et du rejet « inné » induit par le missing self, dans le cas d'une transplantation rénale. $A$. Le dogme actuel est que les rejets du greffon sont déclenchés par la reconnaissance des alloantigènes - i.e. les molécules du complexe majeur d'histocompatibilité (CMH) du donneur - par le système immunitaire adaptatif : les lymphocytes T et B du receveur. Selon les mécanismes effecteurs en cause dans la destruction du greffon, on distingue : (1) les rejets cellulaires, qui résultent de l'infiltration du greffon par les lymphocytes T cytotoxiques détruisant les cellules épithéliales tubulaires dans le cas d'une transplantation rénale (prise ici comme exemple), et (2) les rejets humoraux. Ces derniers sont dus aux anticorps spécifiques du donneur produits par les plasmocytes dans la moelle osseuse du receveur, mais qui sont séquestrés dans la circulation du fait de leur taille. La fixation de ces anticorps à leurs cibles exprimées par les cellules endothéliales de la microcirculation du greffon peut déclencher l'activation de la voie classique du complément ou recruter des effecteurs de l'immunité innée, notamment les lymphocytes NK (natural killer), ce qui se traduit par des signes d'inflammation microvasculaire à l'analyse histopathologique d'un fragment biopsique du greffon. B. Les cellules endothéliales du greffon, qui proviennent du donneur et n'expriment pas les molécules CMH-I du receveur, sont incapables de délivrer les signaux inhibiteurs aux lymphocytes NK circulants du receveur. Cette situation de missing self induit l'activation des lymphocytes NK et la mise en place d'une inflammation microvasculaire responsable d'un rejet chronique vasculaire «inné ». 
notamment pour les molécules du CMHI. Nous avons donc émis l'hypothèse que, lorsque les cellules endothéliales du greffon expriment des molécules du CMH-I incapables de se lier aux KIR inhibiteurs des lymphocytes NK du receveur, ces derniers peuvent s'activer par «missing self» (Figure 1B). En accord avec cette hypothèse, le typage génétique des molécules du CMH-I des paires donneur/receveur dans notre groupe de patients a révélé que ceux qui présentaient une inflammation microvasculaire du greffon sans anticorps spécifiques du donneur présentaient plus souvent une situation de « missing self» [9].

Cependant, un «missing self» génétique n'est pas une condition suffisante pour déclencher un rejet chronique vasculaire puisque certains patients avec « missing self » à l'analyse génétique ne présentaient pas d'inflammation microvasculaire du greffon. Pour répondre au «missing self», les lymphocytes NK doivent être pré-activés par des signaux de danger. Deux situations peuvent jouer ce rôle en transplantation d'organes: les lésions d'ischémie- reperfusion du greffon associées au processus de transplantation et les infections virales (Figure IB). Dans notre groupe de patients, ceux qui ne présentaient pas d'inflammation microvasculaire du greffon avaient effectivement une durée d'ischémie du greffon plus courte et moins d'infections virales (notamment à cytomégalovirus) [9]. Une explication alternative (n'excluant pas l'hypothèse précédente) est liée au fait que l'expression des KIR inhibiteurs par les lymphocytes NK est stochastique. II est donc théoriquement possible qu'un receveur présentant une situation de « missing self » avec son greffon et dont les lymphocytes NK ont été correctement pré-activés, ne développe pas de lésions microvasculaires parce que la population de lymphocytes NK capable de percevoir le «missing self » sur les cellules endothéliales du greffon, c'est-à-dire exprimant le KIR inhibiteur concerné, est numériquement trop faible.
Rejet chronique vasculaire induit par « missing self 》: un rejet «inné 》

Afin de valider nos observations cliniques, nous avons d'abord réalisé des co-cultures de cellules endothéliales et de lymphocytes NK humains. Ce modèle expérimental nous a permis de confirmer que les lymphocytes NK, préalablement activés par l'interleukine-2, peuvent «dégranuler» en réponse à un « missing self» et détruire les cellules endothéliales. II nous a également permis de confirmer l'importance de la voie de signalisation mTOR pour l'activation des lymphocytes NK par «missing self » [9].

Nous avons ensuite procédé à des transplantations cardiaques hétérotopiques chez des souris C57BL/6 en utilisant des greffons provenant de souris $\mathrm{C} 57 \mathrm{BL} / 6$ invalidées pour le gène codant la $\beta 2$ microglobuline (qui stabilise l'expression des molécules du $(M H-I)$, qui sont dépourvues de molécules du CMH-I à la surface de leurs cellules. Dans ce modèle expérimental où le fond génétique des souris donneuse et receveuse est identique, aucune réponse alloimmune n'est possible chez le receveur, notamment la production d'anticorps spécifiques du donneur. Ce modèle expérimental a permis de confirmer nos observations cliniques: des lésions microvasculaires du greffon ont été observées seulement lorsqu'une pré-activation des lymphocytes NK du receveur était déclenchée par ischémie/reperfusion ou par l'administration d'un agoniste du Toll-like receptor 3 (TLR3) afin de simuler une infection virale. En revanche, ces préactivations ne déclenchaient aucune lésion lorsque le greffon provenait de souris témoins exprimant les molécules $\mathrm{du} \mathrm{CMH}-\mathrm{I}$. Le rejet chronique vasculaire était bien dépendant des lymphocytes NK puisqu'il était possible de prévenir l'apparition des lésions microvasculaires d'inflammation du greffon en traitant préalablement les receveurs avec un anticorps monoclonal déplétant les lymphocytes NK [9].
Une piste thérapeutique pour empêcher le rejet chronique vasculaire induit par « missing self »

Des inhibiteurs de mTOR (mechanistic target of rapamycin) ont été développés et mis sur le marché des médicaments dans les années 2000 pour la prévention des rejets du greffon. Initialement conçus comme une alternative aux inhibiteurs de la calcineurine, néphrotoxiques, les inhibiteurs de mTOR sont encore peu utilisés en raison d'un profil de tolérance médiocre. Nous avons comparé les effets de ces deux traitements immunosuppresseurs disponibles sur le marché dans notre modèle expérimental murin. Tandis que l'administration d'inhibiteurs de la calcineurine (ciclosporine) n'avait, comme attendu, aucun effet sur le développement des lésions inflammatoires microvasculaires du greffon cardiaque, les souris traitées avec un inhibiteur de mTOR (sirolimus) ne présentaient presque aucune lésion de rejet chronique vasculaire du greffon [9].

Les résultats de notre étude montrent que l'activation des lymphocytes NK par «missing self» peut déclencher le développement de lésions microvasculaires du greffon, qui conduisent à sa destruction par rejet chronique vasculaire sans l'intervention du système immunitaire adaptatif du receveur (Figure 1B) [9]. L'identification de ce nouveau type de rejet «inné » est importante pour les patients greffés car une option thérapeutique est peut-être déjà disponible. $\diamond$

Missing self-induced NK cell activation promotes "innate" chronic vascular rejection of transplanted organs

\section{REMERCIEMENTS}

O.T. est soutenu financièrement par l'Agence nationale de la recherche (ANR-16-CE17-0007-01), la Fondation pour la recherche médicale (PME20180639518) et l'Établissement français du sang. A.K. est soutenue par l'Inserm (poste d'accueil 2015/1239 / BT), les Hospices civils de Lyon et la Fondation du Rein.

\section{LIENS D'INTÉRÊT}

Les auteurs déclarent n'avoir aucun lien d'intérêt concernant les données publiées dans cet article. 


\section{RÉFÉRENCES}

1. Gaston RS, Cecka JM, Kasiske BL, et al. Evidence for antibody-mediated injury as a major determinant of late kidney allograft failure. Transplantation 2010 ; $90: 68-74$.

2. Chen $C-C$, Pouliquen $\varepsilon$, Broisat $A$, et al. Endothelial chimerism and vascular sequestration protect pancreatic islet grafts from antibody-mediated rejection. J Clin Invest 2018 ; 128 219-32.

3. Sicard A, Ducreux S, Rabeyrin M, et al. Detection of C3d-binding donor-specific anti-HLA antibodies at diagnosis of humoral rejection predicts renal graft loss. J Am Soc Nephrol 2015 ; 26 : 457-67.

4. Hirohashi T, Uehara S, Chase CM, et al. Complement independent antibody-mediated endarteritis and transplant arteriopathy in mice. Am J Transplant $2010 ; 10: 510-7$

5. Hirohashi T, Chase CM, Della Pelle P, et al. A novel pathway of chronic allograft rejection mediated by NK cells and alloantibody. Am J Transplant 2012; 12 : 313-21.

6. Bachelet T, Couzi L, Pitard V, et al. Cytomegalovirusresponsive $\gamma \delta$ T cells: novel effector cells in antibodymediated kidney allograft microcirculation lesions. J Am Soc Nephrol 2014 ; 25 : 2471-82.
7. Yazdani S, Callemeyn J, Gazut S, et al. Natural killer cell infiltration is discriminative for antibodymediated rejection and predicts outcome after kidney transplantation. Kidney Int 2019 ; 95 : 188-98.

8. Gupta A, Broin PÓ, Bao Y, et al. Clinical and molecular significance of microvascular inflammation in transplant kidney biopsies. Kidney Int $2016 ; 89: 217-25$

9. Koenig A, Chen C-C, Marçais A, et al. Missing self triggers NK cell-mediated chronic vascular rejection of solid organ transplants. Nat Commun 2019; $10: 5350$.

10. Thielens A, Vivier $\varepsilon$, Romagné F. NK cell MHC class I specific receptors (KIR): from biology to clinical intervention. Curr Opin Immunol 2012 ; 24 : 239-45. 